\title{
Cross-Correlation-Based Identification of Rotating Sources
}

\author{
W. R. Graham * \\ University of Cambridge, Cambridge, $U K$
}

\begin{abstract}
Noise-source identification using microphone arrays has become well-established in windtunnel testing. When the sources are moving, the accepted approach is first to 'de-Dopplerize' the microphone signals, producing estimated source signals for subsequent analysis. A straightforward time-domain average of these signals is vulnerable to flow-noise contamination, so an alternative approach is necessary for wind-tunnel applications. This method is equivalent to the basic, 'diagonal-removal', cross-spectral technique employed with fixed sources, but, unlike the fixed-source case, cannot be generalized to account simultaneously for multiple sources. The alternative presented here, which works with the time-domain cross-correlations between microphones, is not subject to this limitation. It is illustrated using simulated data from an example rotating-source configuration, and the results are compared against a de-Dopplerization-based analysis. For three equal-strength sources, the current method eliminates the contamination evident in de-Dopplerization estimates. Equally, it is able to locate and accurately characterize weak secondary sources that are masked by primary-source contributions in de-Dopplerization beamforming maps. Hence it extends the option of higher fidelity wind-tunnel beamforming, currently only available for fixed sources, to rotating-source configurations.
\end{abstract}

\section{Nomenclature}

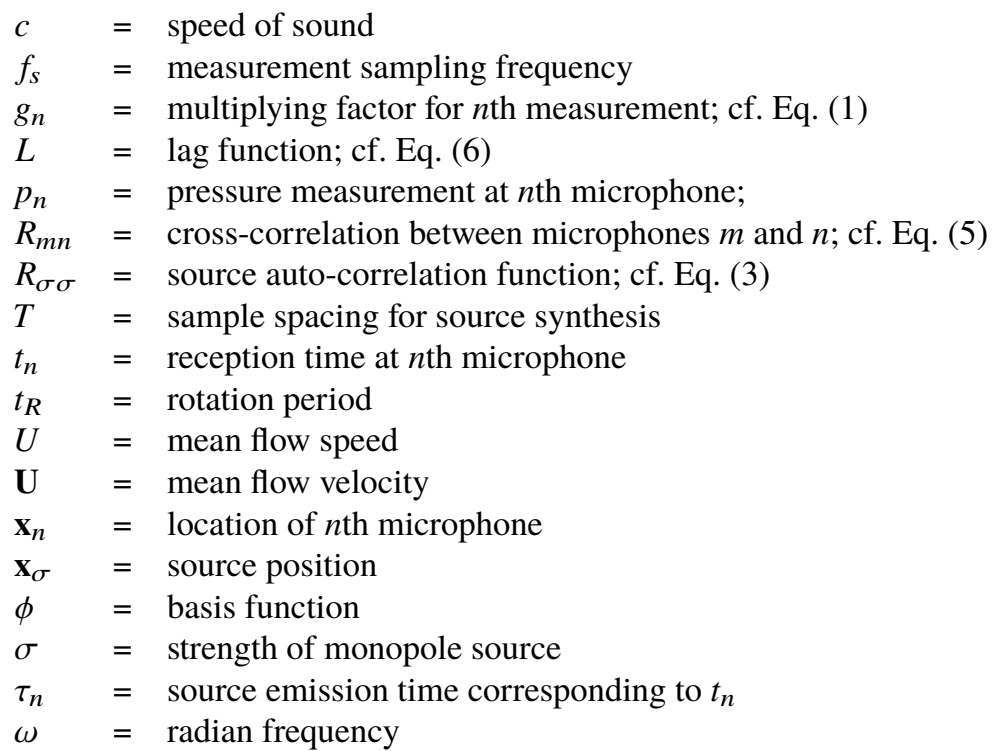

\section{Introduction}

B EAMForming - the identification of source characteristics from the signals received by an array of microphones $\mathbf{B}_{\text {is now routinely employed in the wind-tunnel environment [1, 2]. Typically, a frequency-domain source description }}$ is sought, via the estimated cross-spectra between microphones. This allows the benefits of an array configuration — spatial discrimination and rejection of extraneous noise — to be realized [3]. For a closed-section wind tunnel with flush-mounted wall microphones there is an additional advantage: given sufficient averaging, the cross-spectrum

*Senior Lecturer, Department of Engineering, Trumpington Street, Cambridge, CB2 1PZ; Senior Member AIAA. 
estimates are insensitive to unsteady pressures associated with flow turbulence, because such pressures are correlated over much smaller distances than acoustic disturbances [3].

Given the cross-spectra, various algorithms for source identification are available. The most basic simply seeks the single source at a given location that best accounts for the cross-spectra, and builds a source map by repeating this process over many locations [3]. Contamination of each estimate due to extraneous contributions is limited solely by the array's discrimination ability. More advanced approaches make allowance in their formulation for the presence of other sources. In principle, it is possible to write the cross-spectra in terms of the (unknown) contributions from all sources under consideration, thereby avoiding the mutual contamination problem [4]. In practice, the associated beamforming calculation is numerically prohibitive, and the solution must be approximated by an iterative approach [4, 5].

If the sources are in motion relative to the array, the problem is complicated by the well-known Doppler frequency shift. This implies that microphone signal components at a given frequency are no longer directly related to the source components at that frequency. Moreover, the microphone signals are now not statistically stationary, even if the source is. Analyses based on conventional estimates of microphone cross-spectra are thus precluded. The solution in current use $[6-8]$ is to generate source-signal estimates by 'de-Dopplerizing' the microphone records. Averaging these estimates attenuates contributions from other sources, and provides a time-domain signal for further processing. Apart from including amplitude scaling factors associated with the source/microphone distances, this approach corresponds to the classical 'delay-and-sum' technique. It is carried out separately for each presumed source, and hence, like basic cross-spectral beamforming, relies on the array alone for discrimination. Unlike cross-spectral beamforming, however, it cannot be generalized to account for multiple sources.

The delay-and-sum algorithm is also vulnerable to flow-turbulence pressure contributions, which remain in the averaged signal, and thus also its spectrum [7]. They are attenuated by averaging, but often not sufficiently (because the average is only over the number of microphones in the array). However, their expected contribution can be eliminated by subtracting the mean of the individual-estimate autospectra, suitably scaled, from the autospectrum of the averaged source estimate (the 'alternative reconstruction' [7]). It is straightforward to show that this formulation corresponds to forming all the cross-spectra between source-estimate pairs and averaging them, i.e. it is directly analogous to cross-spectral beamforming. It carries a price, though: the spectral analysis implicitly assumes that the source signal is stationary. In practice, slow variation in the source parameters ('quasi-stationarity') can be handled by controlling the length of data blocks, but this implies a limit on the frequency resolution available.

Here, an alternative method not subject to these restrictions is introduced. It employs the (time-domain) microphone cross-correlation functions, with non-stationarity explicitly accounted for. These quantities can be written in terms of source auto-correlation functions, meaning that simultaneous or iterative identification of multiple sources becomes possible. The shift to this formulation also removes the need to assume that the sources are statistically stationary (because the source auto-correlations are generalized in the same way as the microphone cross-correlations). On the other hand, it implies onerous computer-memory requirements. Nonetheless, as will be shown, this issue can be mitigated to the extent that implementation on a present-day desktop computer becomes possible.

A configurational limitation should also be noted. To estimate the cross-correlations, the data must be averaged in an ensemble sense. The sources must therefore repeat their trajectories many times, implying that the majority of viable practical applications will involve rotating devices. This, however, is not a significant constraint in the context of wind-tunnel tests, where any source motion is almost invariably rotational. Rotation will thus henceforth be assumed.

The paper consists of two main parts. The first, Section II introduces the formulation and explains how it can be solved to characterise the sources. The second, Section III] presents simulated results for a specific source/measurement configuration. For comparison purposes, it also includes results from de-Dopplerization-based source identification via the flow-noise-insensitive 'alternative reconstruction' of the source spectrum [7, 8], henceforth referred to as 'ROSI'.

\section{Formulation}

\section{A. Microphone cross-correlations}

For simplicity, the arguments here will be developed on the basis of a monopole source. In this case [7], the signal $p_{n}\left(t_{n}\right)$ received by the $n$th microphone at time $t_{n}$ can be written as

$$
p_{n}\left(t_{n}\right)=g_{n}\left(\tau_{n}\right) \sigma\left(\tau_{n}\right),
$$

where $\tau_{n}$ is the source emission time associated with $t_{n}$, and $\sigma$ is the source strength. For more complex sources, the multiplying factor $g_{n}$ becomes an operator acting on $\sigma$, but the points to follow remain valid. 
Consider now the correlation between two microphone signals. From Eq. (1) this is given by

$$
\overline{p_{m}\left(t_{m}\right) p_{n}\left(t_{n}\right)}=g_{m}\left(\tau_{m}\right) g_{n}\left(\tau_{n}\right) R_{\sigma \sigma}\left(\tau_{m}, \tau_{n}\right),
$$

where $R_{\sigma \sigma}$ is the source auto-correlation function:

$$
R_{\sigma \sigma}\left(\tau_{m}, \tau_{n}\right)=\overline{\sigma\left(\tau_{m}\right) \sigma\left(\tau_{n}\right)}
$$

Equation (2) confirms the earlier assertion that the microphone cross-correlations are not statistically stationary, even if the source itself is. However, because the source finds itself in the same position with the same statistical properties once per revolution, they are at least cyclo-stationary (i.e. periodic with the source revolution time $t_{R}$ ). Thus

$$
\overline{p_{m}\left(t_{m}+k t_{R}\right) p_{n}\left(t_{n}+k t_{R}\right)}=\overline{p_{m}\left(t_{m}\right) p_{n}\left(t_{n}\right)}
$$

for $k$ integer. In the applications envisaged here, it will be more convenient to express the cross-correlations as functions of the variables $t_{m}$ and $t_{n}-t_{m}$, so the cross-correlation function between the signals measured at microphones $m$ and $n$ is defined as

$$
R_{m n}\left(t_{m}, t_{n}-t_{m}\right)=\overline{p_{m}\left(t_{m}\right) p_{n}\left(t_{n}\right)}
$$

This quantity is periodic in $t_{m}$ for fixed $t_{n}-t_{m}$.

The available data will consist of discrete signal measurements, taken at regular time intervals. The cross-correlation functions will thus likewise be known for discrete, regularly spaced, values of the arguments $t_{m}$ and $t_{n}-t_{m}$. The associated source times $\tau_{m}, \tau_{n}$ in the source auto-correlation function $R_{\sigma \sigma}$ will not be regularly spaced, and each microphone pair will give rise to a different set. Hence a parametric representation for $R_{\sigma \sigma}\left(\tau_{m}, \tau_{n}\right)$, allowing it to be evaluated for arbitrary arguments, is needed. This conclusion applies even more strongly if the simple proportionality relationship of Eq. (1) is inapplicable. For example, dipole sources lead to microphone cross-correlations involving derivatives of the source auto-correlation, which can only be evaluated consistently via some form of parameterization.

\section{B. Source auto-correlation parameterization}

Every instance of Eq. (2) provided by the microphone signals will give rise to an equation for the (unknown) parameters used to define $R_{\sigma \sigma}\left(\tau_{m}, \tau_{n}\right)$. For typical microphone numbers and data sampling rates, there will be sufficient equations that storage and computational costs are potentially unmanageable. Therefore, it is necessary to minimize the size of the parametric representation as much as possible.

The first step is to express the source auto-correlation in terms of the mean and difference of its arguments. As it has the same periodicity properties as the microphone cross-correlations, it can be written in the form

$$
R_{\sigma \sigma}\left(\tau_{m}, \tau_{n}\right)=L\left(\tau_{n}-\tau_{m}\right)+\sum_{k=1}^{K}\left[\cos \left(k \pi \frac{\tau_{m}+\tau_{n}}{t_{R}}\right) C_{k}\left(\tau_{n}-\tau_{m}\right)+\sin \left(k \pi \frac{\tau_{m}+\tau_{n}}{t_{R}}\right) S_{k}\left(\tau_{n}-\tau_{m}\right)\right] .
$$

If the source is statistically stationary, $C_{k}$ and $S_{k}$ are zero and the summation disappears. Otherwise, one would still expect the dependence on $\left(\tau_{m}+\tau_{n}\right) / 2$ to be relatively slow (i.e. to be on the scale of the revolution time). Hence the required number of harmonics, $K$, is likely to be small.

The functions of $\tau_{n}-\tau_{m}$, on the other hand, will vary on a time-scale associated with the bandwidth of the source signal. For a broadband source, this will typically be many times the revolution frequency. Then $L(\tau)$ (for example) will decay rapidly away from $\tau=0$, and a spectral parameterization would be inefficient. An alternative is a basis-function expansion of the form

$$
L(\tau)=\sum_{j} l_{j} \phi\left(f_{s} \tau-j\right)
$$

This is well suited to the local nature of the dependence, because the basis function $\phi$ can be of finite, and short, extent. Then the total number of coefficients required will be proportional only to the overall extent of $L(\tau)$. Equally, just a small number of the coefficients $l_{j}$ will contribute to a given evaluation of $L(\tau)$, so each instance of Eq. (2) will be sparse in terms of the unknowns. The specific choice of basis function will be discussed subsequently, in Section III. 


\section{Source identification}

Apart from its efficiency, a crucial feature of the parameterization proposed here is that it is linear in the unknown coefficients of the basis-function expansions. For example, if the source is statistically stationary, Eqs. (5), (2), (6) and 77) combine to give

$$
\sum_{j}\left\{g_{m}\left(\tau_{m}\right) g_{n}\left(\tau_{n}\right) \phi\left(f_{s}\left[\tau_{n}-\tau_{m}\right]-j\right)\right\} l_{j}=R_{m n}\left(t_{m}, t_{n}-t_{m}\right) .
$$

The multiple instances of this expression available from the data will constitute an over-determined set of linear equations for the $l_{j}$ s (and, in the general case, the equivalent coefficients specifying $C_{k}, S_{k}$ ), which can be solved in a least-squares sense. Thus, in principle, the required quantity $-R_{\sigma \sigma}\left(\tau_{m}, \tau_{n}\right)-$ can be found.

The discussion thus far has assumed a single source. However it also applies when there are multiple uncorrelated sources. In this case, the right-hand side of Eq. (2) becomes a sum of source auto-correlation contributions, each parameterized with its own coefficient set. In terms of Eq. (8), the right-hand side now has multiple groups of $l_{j}$ s, one per source. No change to the solution approach is required and thus, as claimed previously, all the auto-correlations can be estimated simultaneously. Equally, the contribution of any individual source auto-correlation estimate to the microphone cross-correlations can be identified and removed, leaving modified cross-correlations amenable to the same analysis. This opens the door to iterative source identification.

If the sources are correlated, the current method remains applicable in principle, but its implementation may become more complicated. The right-hand side of Eq. (2) now additionally contains source cross-correlations, which again require parameterization. However, the associated lag functions will not necessarily peak at zero time delay. A greater time-delay range could be allowed for, but this would reduce the efficiency of the parameterization. Alternatively, the time-delay offset could be identified in a preliminary calculation. These issues are beyond the scope of the current paper.

\section{Algorithm description}

Here the computational steps needed to implement the formulation set out above are summarized. For definiteness, they are presented with reference to Eq. (8), but the same process applies for the general case.

- Estimate the microphone-signal cross-correlations. Here, because of cyclo-stationarity, the ensemble average required by Eq. (5) can be replaced by averaging over revolutions. For each argument pair $\left(t_{m}, t_{n}-t_{m}\right)$ there is, by virtue of Eq. (4), one sample per revolution. The upshot is a dataset of cross-correlation levels at uniformly spaced values of $t_{m}$ (covering the revolution period) and $t_{n}-t_{m}$ (covering the range needed to capture significant levels).

- Precalculate the source times $\tau_{m}$ and multiplying factors $g_{m}\left(\tau_{m}\right)$ corresponding to the set of $t_{m}$. This information is required for each microphone.

- Assemble the equation set. Within a loop over microphone pairings, implement Eq. (8) for each available value of $R_{m n}$.

- Solve the equations. Find the values of the coefficients $l_{j}$ that minimize the sum of squared errors in the instances of Eq. (8).

\section{E. Assessment}

Setting out the current approach in principle will be of little use if it is not feasible in practice. If it is, one also wants to know how well it performs. It is hard to envisage useful answers to these questions without empiricism. Both depend strongly on the details of the acoustic array and on the sound sources under investigation. Therefore, the remainder of the paper will consider numerical simulations of a specific configuration.

The characterization of performance will be made with reference to the ROSI method. In particular, the potential benefits of the current formulation's additional capabilities - simultaneous identification of multiple sources, and iterative source-by-source analysis — will be addressed. Practical implementation issues will also be discussed.

\section{Simulations}

\section{A. Configuration}

The physical configuration used for the simulations is motivated by wind-tunnel experiments on a vertical-axis wind-turbine model [9]. The geometry of the experimental set-up is shown schematically in Fig. 1] An acoustic array is flush-mounted in the tunnel floor, and the wind-turbine model is installed horizontally, with its rotation axis $0.61 \mathrm{~m}$ 
above the array plane. Details of the array are given in Ref. [10]; it consists of 48 microphones arranged on concentric ellipses with maximum extent $1.78 \mathrm{~m}$ (streamwise) $\times 0.89 \mathrm{~m}$. The origin of coordinates is taken at the array center, with $x$ in the streamwise direction and $z$ vertically upwards. The turbine-model blade radius is $0.265 \mathrm{~m}$; in these simulations, primary blade sources at an axial position $y=0.1 \mathrm{~m}$ will be considered. The flow speed, $U$, is set at $5 \mathrm{~m} / \mathrm{s}$ and the turbine rotation rate at 12 rev/s. Finally, the speed of sound is taken to be $340 \mathrm{~m} / \mathrm{s}$.

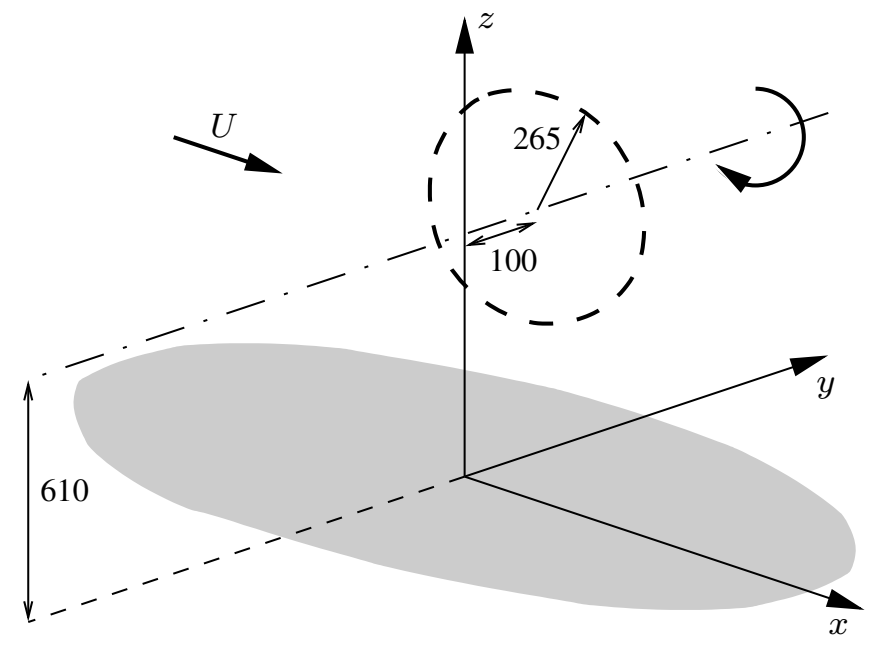

Fig. 1 The physical configuration employed for the simulations (dimensions in mm), with acoustic array shaded. The dash-dot line indicates the source rotation axis, and the thick dashes show the circular source path.

\section{B. Signal generation and processing}

\section{Transmission factors}

The simulations employ monopole sources, to which the formulation introduced in Section $\Pi$ for the pressure at the $n$th microphone $-p_{n}\left(t_{n}\right)=g_{n}\left(\tau_{n}\right) \sigma\left(\tau_{n}\right)$ - applies. In particular, for a source with path $\mathbf{x}_{\sigma}(\tau)$ in a flow with velocity U, Sijtsma [8] gives

$$
g_{n}\left(\tau_{n}\right)=-\frac{1}{4 \pi} \frac{1}{\left|\mathbf{r}_{n}\right|-\mathbf{r}_{n} \cdot\left[\dot{\mathbf{x}}_{\sigma}\left(\tau_{n}\right)-\mathbf{U}\right] / c},
$$

with

$$
\mathbf{r}_{n}=\mathbf{x}_{n}-\mathbf{x}_{\sigma}\left(\tau_{n}\right)-\mathbf{U}\left(t_{n}-\tau_{n}\right),
$$

in which $\mathbf{x}_{n}$ is the microphone position and

$$
t_{n}-\tau_{n}=-\frac{\mathbf{U} \cdot\left(\mathbf{x}_{n}-\mathbf{x}_{\sigma}\right)}{\left(c^{2}-U^{2}\right)}+\frac{\sqrt{\left[\mathbf{U} \cdot\left(\mathbf{x}_{n}-\mathbf{x}_{\sigma}\right)\right]^{2}+\left(c^{2}-U^{2}\right)\left|\mathbf{x}_{n}-\mathbf{x}_{\sigma}\right|^{2}}}{\left(c^{2}-U^{2}\right)} .
$$

Hence, given the emission time $\tau_{n}$, the microphone pressure can be found from the source signal. Note, however, that Eq. (11) cannot be explicitly inverted for $\tau_{n}$ as a function of $t_{n}$, so iteration is required in order to obtain microphone signals at specified instants.

\section{Synthetic data}

Equation (2) relates the microphone cross-correlations directly to the source auto-correlation. Hence it would be possible to simulate the identification method proposed here purely on this basis. However, the ROSI algorithm starts from microphone-signal time histories. To enable comparison between the approaches, therefore, a stochastic source signal with known statistics must be generated. 
For clarity and simplicity, a statistically stationary source signal is specified. This allows the underlying assumption in the ROSI method to be met exactly, so (as the transmission factors are definitively known here) the ROSI estimate for a single-source case should be accurate to within statistical uncertainty. Meanwhile, the associated microphone signals remain non-stationary, thus providing a legitimate test case for the current approach.

Practical methods for stochastic signal synthesis produce uniformly spaced samples. These will correspond to non-uniformly spaced arrival times at the microphones. Interpolation of the synthesized source signal will thus be necessary, and this must not introduce significant error if the process responsible for the simulated microphone signals is to be assumed known and comparable against the outputs of the identification algorithms. The detail implications of this point are discussed in Appendix A, which describes how the simulated signals are generated. The upshot is a source signal effectively band-limited to below $11.8 \mathrm{kHz}$, and associated microphone signals with interpolation errors under $0.01 \%$. The latter are sampled at $24.6 \mathrm{kHz}$ (2048 points per revolution), which provides sufficient margin to cover Doppler frequency shifts.

Results from the source-identification calculations will be assessed over frequencies from 0 to $8 \mathrm{kHz}$. The source spectrum in this range is plotted in Fig. 2

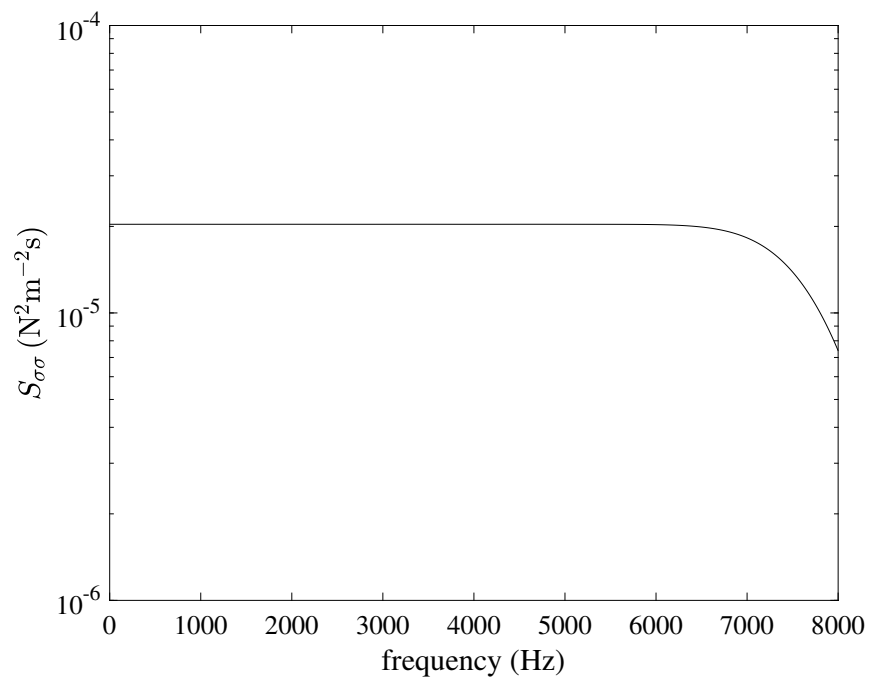

Fig. 2 Spectrum of the simulated source signal (cf. Eqs. (14) and (15)).

\section{Cross-correlation functions}

Recall that the cross-correlation between microphones $m$ and $n$ is periodic in the sense that it takes the same value for all time pairs $\left(t_{m}+k t_{R}, t_{n}+k t_{R}\right)$, with $k$ integer. Hence the number of unique time pairs is $2048 \times 2048$, and for each there is one sample of $p_{m} p_{n}$ per source revolution. These samples can be averaged to form the cross-correlation estimate.

In practice, calculating the estimate for every time pair of every microphone pair would be computationally punitive. Fortunately, this is unnecessary; the fundamental assumptions on the source auto-correlation set out in Section II imply that $R_{m n}\left(t_{m}, t_{n}-t_{m}\right)$ will only be non-negligible for a restricted range of the lag $t_{n}-t_{m}$. This expectation is confirmed by the single-source example plotted in Fig. 3. whose contours show that the region of significant correlation is concentrated around a specific locus: the line where $t_{m}$ and $t_{n}$ correspond to the same source emission time. In this case the lag variation due to the locus adds approximately $1.5 \mathrm{~ms}$ to the maximum source lag $(1.4 \mathrm{~ms}$, given the filter defined in Appendix A). The active lag range is thus $4.3 \mathrm{~ms}$, compared to a rotation period of $83.3 \mathrm{~ms}$. Additional sources on the same path will contribute identical patterns, offset in $t_{m}$.

The relevant lag range can be established in advance of the calculation, given the source path and the maximum significant source-auto-correlation lag. This process naturally leads to a tailored lag range for each microphone pair, further reducing computation and storage requirements. In practice, of course, it will usually be necessary to seek sources on other paths too, and in Section III.F this will be done to assess discrimination capability. The current implementation of lag-range specification thus considers four possible source paths, at the axial $(y)$ locations closest to, 


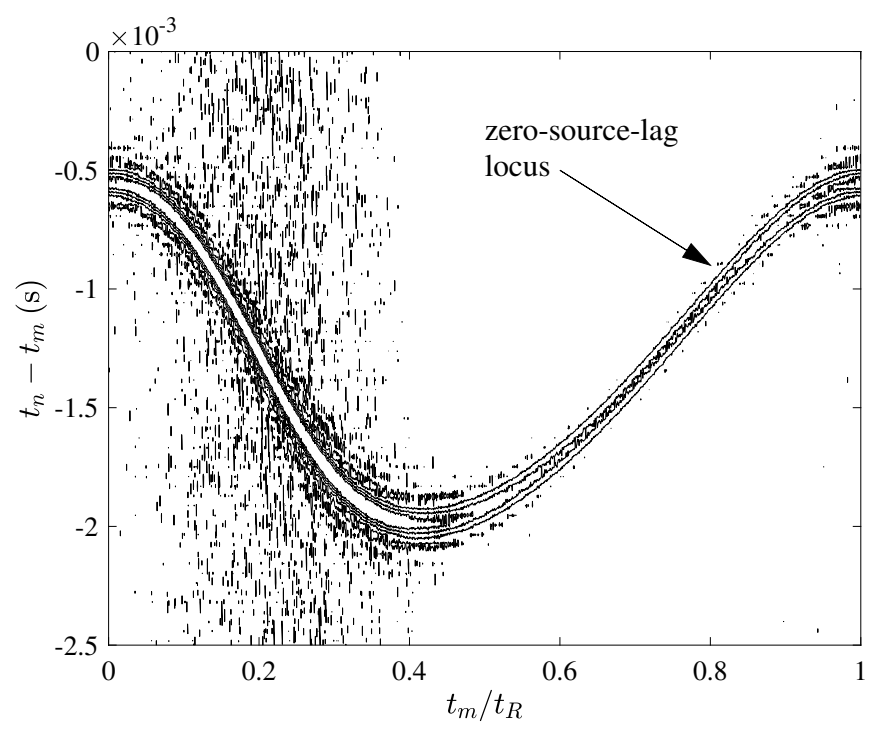

Fig. 3 Cross-correlation contours (single source, 400 averages) for microphones with $\mathbf{x}_{m}=(0.89,0,0) \mathrm{m}$, $\mathbf{x}_{n}=(0,0,0) \mathrm{m}$. Levels from $-1.5 \times 10^{-3}$ to $2.5 \times 10^{-3}$ with $1 \times 10^{-3}$ spacing.

and furthest from, each microphone in the pair under consideration. The associated relevant-lag-range increases are slight. The upshot is that $257 \times 10^{6}$ cross-correlation values are calculated, compared to the $4730 \times 10^{6}$ that could be. (Microphone auto-correlations are not used, because of the flow-noise contamination that would be present in a real application.)

\section{Cross-correlation downsampling}

Recall that each cross-correlation value potentially gives rise to an instance of Eq. (8). Thus further reduction in their number is desirable. It can be achieved by down-sampling the dependence on $t_{m}$. To see that this is feasible, consider Fig. 4. which shows the spectral content of Fig. 3 across $t_{m}$ for lags between -2.0 and $-0.5 \mathrm{~ms}$. The 2048-sample data lines contain Fourier orders $k$ up to 1024, but the useful information is restricted to those below about 50. Hence only about 100 samples are needed in this case, and the number of equations can be reduced approximately 20 -fold. Other microphone pairs, with less variation in $t_{n}-t_{m}$ along the zero-source-lag locus, show even greater potential.

Down-sampling is implemented by frequency-domain filtering, followed by an inverse discrete-Fourier-transform of the rotation orders retained. To minimize its impact on the data, the filter is simply a linear taper from unity at the microphone-pair cut-off order to zero at the next power of two. The cut-off order is tailored for each pair, according to the algorithm described in Appendix B. This step reduces the potential number of equations from $257 \times 10^{6}$ to $15.9 \times 10^{6}$.

\section{Source auto-correlation fitting}

Having derived the data whose values appear on the right-hand side of Eq. (8), it is now necessary to specify the basis functions employed in the terms on the left-hand side. This section also describes how relevant instances of Eq. (8) are identified for inclusion in the least-squares problem.

\section{Parameterization}

The basis functions $\phi$ parameterize the lag dependence of the source auto-correlation according to Eqs. (6) and (7). They must be short, to make the least-squares equation matrix manageable. Equally, they should be as smooth as possible, so that discontinuities in $L(\tau)$ and its derivatives are insignificant.

The requirements of smoothness and compactness are optimized by 'basis splines' [11]. Here, the cubic member of this family is employed, giving 


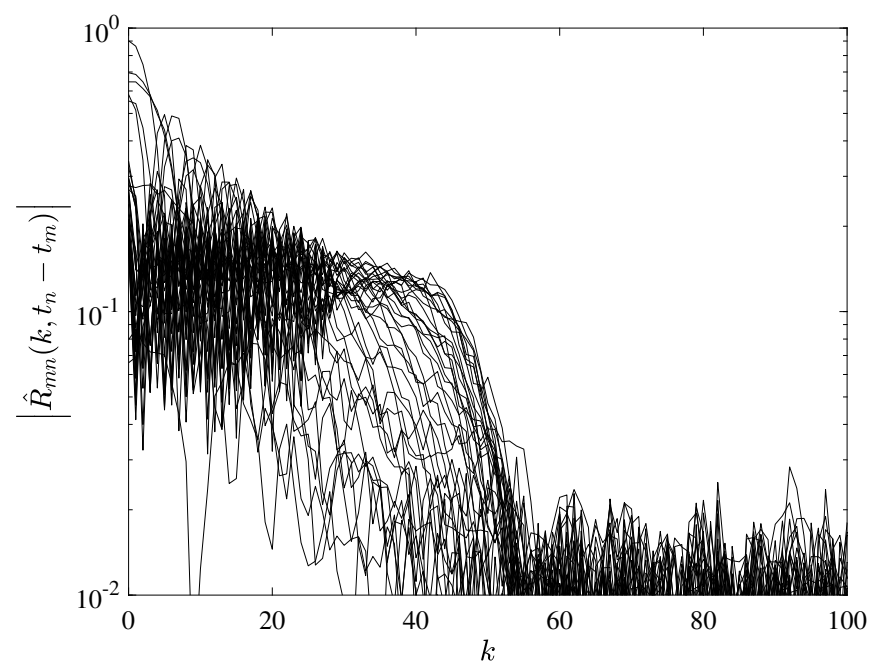

Fig. 4 Discrete Fourier transform $\hat{R}_{m n}\left(k, t_{n}-t_{m}\right)=\sum_{j} R_{m n}\left(j / f_{s}, t_{n}-t_{m}\right) \mathrm{e}^{-\mathrm{i} 2 \pi j k / 2048}$ of Fig. 3 , for $t_{n}-t_{m}$ between -2.0 and $-0.5 \mathrm{~ms}$.

$$
\begin{aligned}
\phi(u) & =\frac{2}{3}-u^{2}+\frac{1}{2}|u|^{3}, \quad 0 \leq|u|<1 \\
& =\frac{4}{3}-2|u|+u^{2}-\frac{1}{6}|u|^{3}, \quad 1 \leq|u|<2 \\
& =0, \quad \text { otherwise. }
\end{aligned}
$$

This implies that only four of the coefficients $l_{j}$ in Eq. (7) contribute to each evaluation of $L$.

The extent of the auto-correlation underlying the source simulation is $\pm 1.4 \mathrm{~ms}$, but it decays rapidly away from zero lag. The fitted auto-correlations derived here cover the range $\pm 0.9 \mathrm{~ms}$. As the lag function is even, they can be characterized by 21 coefficients.

\section{Equation assembly}

Once the source parameterization has been defined, it is possible to identify which data points in the down-sampled cross-correlation set correspond to a lag in the source auto-correlation range, and hence to a usable instance of Eq. (8). The lag evaluation can be expedited by pre-calculating look-up tables of source emission times corresponding to a single revolution of microphone reception times. For the single-source simulation, $6.05 \times 10^{6}$ of the $15.9 \times 10^{6}$ cross-correlation values are relevant.

For each relevant value, the non-zero entries in the equation matrix arise from the indices $j$ such that $\left|f_{s} \tau-j\right|<2$ (cf. Eqs. (7) and (12)). In the single-source case, there are $22.3 \times 10^{6}$ such entries. Thus, as expected, the $6.05 \times 10^{6}$ by 21 matrix is highly sparse, easing storage requirements. Equally, the least-squares solution is not numerically demanding, despite the size of the equation set; here the Matlab 'mldivide' (backslash) function ${ }^{\text {f }}$ is employed. The implementation for multiple sources is a straightforward generalization of the single-source approach, subject to the caveat that some data values become relevant to more than one source.

\section{Convergence}

The first set of identification results that will be presented are for the single-source case. This conforms exactly to the assumptions of both the current method and the ROSI approach, so the only errors should be due to statistical uncertainty. Thus the output serves both as an algorithm check and an empirical assessment of convergence behavior. It is presented in spectral form; to do so, the basis-spline expansion for the source auto-correlation is Fourier-transformed analytically, using the standard results quoted in Ref. [12].

\footnotetext{
*https://uk.mathworks.com/help/matlab/ref/mldivide.html, 29 January 2019.
} 
For the convergence assessment, two reduced-sample cases are analyzed. They have one-half and one-quarter the length of the base data set, meaning that the microphone cross-correlations are formed from 200 and 100 averages respectively. When analyzed with the current method, all three yield results in close agreement with the true source spectrum. Their levels relative to this baseline are plotted in Fig. 55 There is a consistent improvement as the number of averages increases, as one would expect. More surprisingly, the uncertainty is small in comparison to the standard deviation of a periodogram-based spectral estimate using 400 averages without overlap $(0.21 \mathrm{~dB}$, on the basis of the expressions given by Welch [13]).

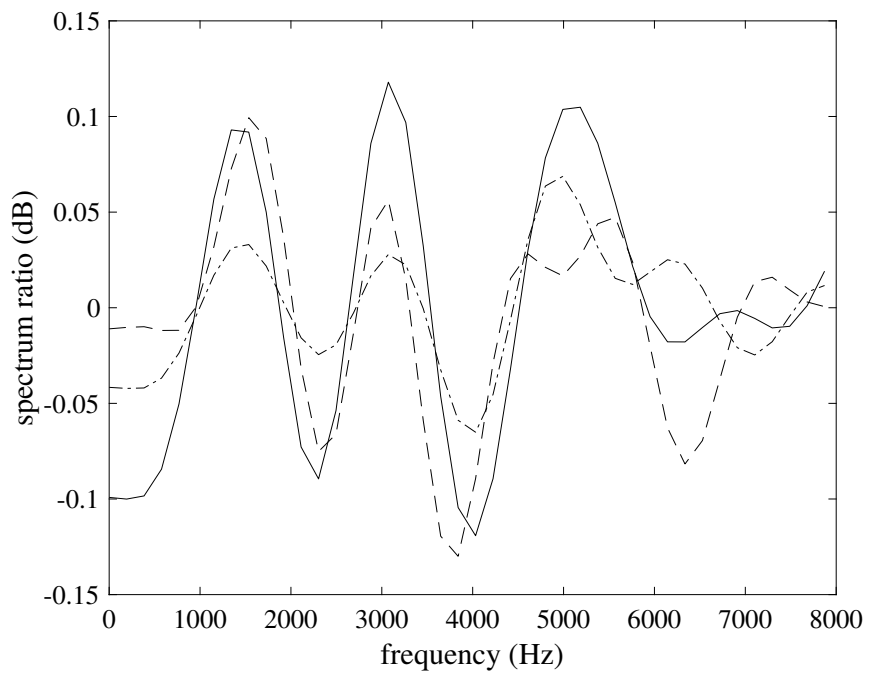

Fig. 5 Source-spectrum estimates, relative to the true level. Single-source simulation, with microphone cross-correlations estimated using $100(-), 200(--)$, and $400(-\cdot-)$ averages.

Next, the 400-average results are compared against the ROSI-based estimate. The ROSI implementation follows Sijtsma et al. [7], except that the interpolation required to produce the estimated source signal associated with each microphone is based on septimic basis splines. To reduce error, the interpolant must be re-sampled at a higher frequency than the data it is based on [12]; here, to allow convenient comparison with true spectrum values, the rate is doubled. This implies that the interpolation attenuates the microphone signal spectra below $8 \mathrm{kHz}$ by at most $0.03 \mathrm{~dB}$, and the aliasing that it introduces in this range is negligible. The estimated source spectra are calculated via standard periodogram averaging [13], with 50\% overlap, Hann window, and block size chosen so the number of averages is 400. The comparison with the result from the cross-correlation-based method is shown in Fig. 6 . The uncertainty is consistent with Welch's $0.22 \mathrm{~dB}$ standard-deviation value for these analysis parameters [13], as it should be if the source signal is recreated without significant error. (No benefit is gained from the array in this case, as each microphone conveys identical information.)

The difference in uncertainty between the two methods warrants discussion. Intuitively, it can be ascribed to the reduced frequency resolution of the cross-correlation fitting approach. A direct fast-Fourier-transform of the fitted source auto-correlation would yield spectrum values approximately every $400 \mathrm{~Hz}$, and the uncertainty in the ROSI-derived spectrum would be greatly reduced if it were smoothed over 'bins' of this extent. However, it is not straightforward to substantiate this explanation on the basis of the fitting equations. These imply a linear dependence of the fitted source auto-correlation on the microphone cross-correlations, whose variance will be commensurate with that of the source-spectrum estimate (for the same number of averages). Hence any equivalent spectral smoothing must arise through the selection of relevant data points and the inversion of the equations. These aspects are difficult to analyze. What can be said is that a ROSI-based estimate with comparable spectral resolution would have access to more averages than the cross-correlation-fitting approach, and would thereby become less uncertain than the one considered here. Equally, the current method converges more rapidly with average number than conventional spectral estimates. 


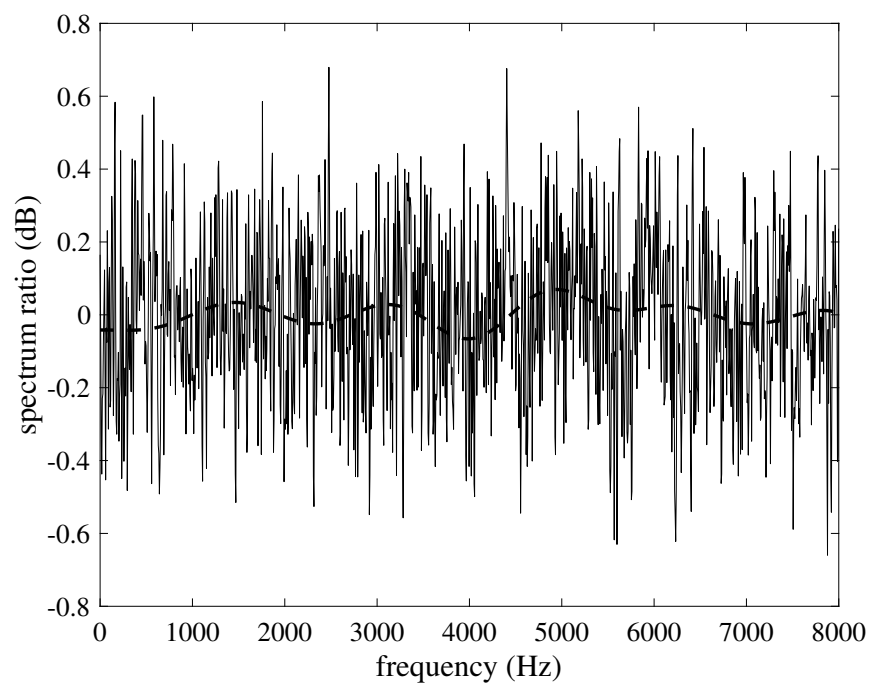

Fig. 6 Source-spectrum estimates based on 400 averages, relative to the true level. Single-source simulation, with estimation via ROSI (-) and current method (- -).

\section{E. Multiple source identification}

Recall that the current, cross-correlation-fitting, method can account for the presence of more than one source. To assess the benefit of this capability, microphone signals for three equi-spaced sources are generated. The sources are uncorrelated, so the expected cross-correlations are simply superpositions of their single-source counterparts. This situation is straightforward to handle in the framework of the current method, so the source spectrum should be identified with the same accuracy as before. In contrast, the ROSI approach requires a separate analysis for each source, in which the other two will contaminate the estimate. The upshot is shown in Fig.77(again relative to the baseline level); the ROSI spectrum is significantly in error at low frequencies, and only settles around the true value at about $3 \mathrm{kHz}$. Even here, oscillations beyond those associated with statistical uncertainty are in evidence. Meanwhile, the cross-correlation-based estimate lies (to within statistical accuracy) at $0 \mathrm{~dB}$, as expected.

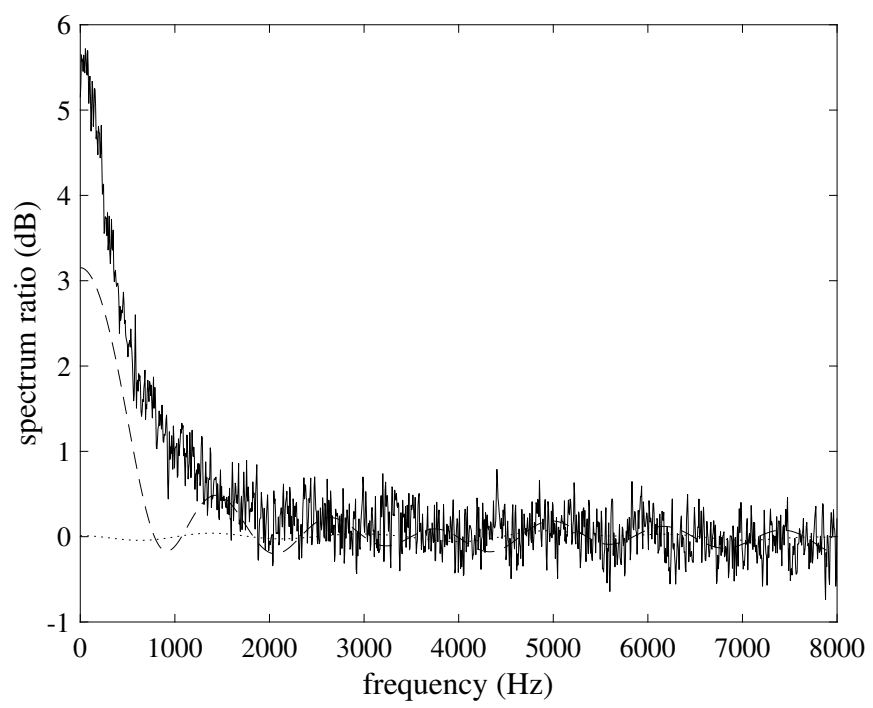

Fig. 7 Source-spectrum estimates, relative to the true level. Three-source case, analyzed with: - , ROSI; $\cdots$, current method, three sources; --, current method, one source. 
Also shown in Fig. 7 is the result of performing a cross-correlation-based fit assuming, like the ROSI calculation, a single source only. This too exhibits contamination error at low frequencies, but, interestingly, to a lesser degree than the ROSI output. The frequency at which it settles around the true value is similar, meaning that there is little to choose between the two estimates above about $2 \mathrm{kHz}$.

The universality of this finding remains unknown. However, both single-source estimates confirm the effectiveness of array measurements in rejecting extraneous contributions. The microphone layout in the simulations was designed to be applicable for frequencies between $650 \mathrm{~Hz}$ and $6.5 \mathrm{kHz}$ [10], and here the spectra are only about $2 \mathrm{~dB}$ in error at worst. For most of the range, they are within $0.25 \mathrm{~dB}$ of the true value. To put these figures in context, a three-fold increase in sound power corresponds to a level shift of $4.8 \mathrm{~dB}$.

\section{F. Spatial discrimination}

So far, it has been assumed that the source positions are known, and the problem is simply to determine their levels. In many practical applications, however, beamforming is also used for source location. The ability of acoustic arrays to discriminate against contributions from sources away from the focus point means that maps of estimated source amplitude on a grid of trial locations typically show maxima at, or close to, true source positions. This property is certainly true of ROSI [7], but needs to be demonstrated for the current method. It is assessed, using the single-source case, in Section III.F.1

When multiple sources are present, interference is possible, to the extent that secondary, less powerful, contributors may not even be locatable via conventional beamforming. With stationary sources, the problem is mitigated by multiple-source formulations, so the same should be true for rotating sources analyzed using the current method. This issue is explored in Sections III.F.2 and III.F.3

\section{Resolution}

Figure 8 shows maps of the source-spectrum amplitude estimated by ROSI for the single-source case at frequencies of $2.4 \mathrm{kHz}$ and $4.8 \mathrm{kHz}$. As expected, the estimates have their maxima at the true location. Also evident is the well-known frequency dependence of the array resolution: the breadth of the maximum is reduced at the higher frequency. Far enough away, the estimates become negative; these unphysical results are indicated by white space.

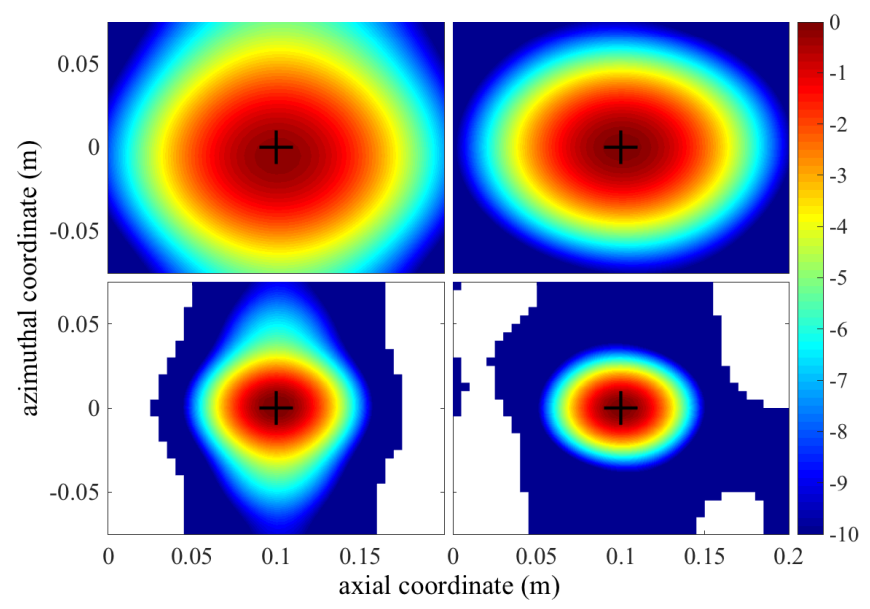

Fig. 8 Estimated spectrum levels, dB relative to true value, for the single-source case. Left: ROSI; right: current method. Top: $2.4 \mathrm{kHz}$; bottom: $4.8 \mathrm{kHz}$. Cross indicates source position.

Beside the ROSI maps are the corresponding plots for the current method. These confirm that it, too, has the expected behavior. Indeed, in this example, the resolution is slightly better at both frequencies, and at $2.4 \mathrm{kHz}$ the peak is more symmetrical with respect to the source position. Nonetheless, in broad terms the performance is similar; as might have been anticipated, the resolution is essentially determined by the array configuration. 


\section{Side-lobe interference}

It is clear that a secondary, weaker, source lying within the beamforming maximum of Fig. 8 is likely to be masked. Less evident is the possibility of interference away from this region. It arises because, even here, there are spurious level estimates ('side-lobes') [2, 3]. For the ROSI algorithm, these are approximately $17 \mathrm{~dB}$ below the true level. Figure 9 shows their impact on the ROSI estimates for a secondary source $0.2 \mathrm{~m}$ from the primary, and $17 \mathrm{~dB}$ weaker. (N.B. The image region is $0.2 \mathrm{~m}$ to the left of that used for Fig. 8 and hence does not include the main source.) At $2.4 \mathrm{kHz}$, there is no clear evidence that the source is present, and the map is dominated by the outer edge of the primary-source peak (the 'main lobe'). At $4.8 \mathrm{kHz}$, the secondary source is visible, but with level approximately $7 \mathrm{~dB}$ below its true value. (Note that the side-lobes are negative in this region.)

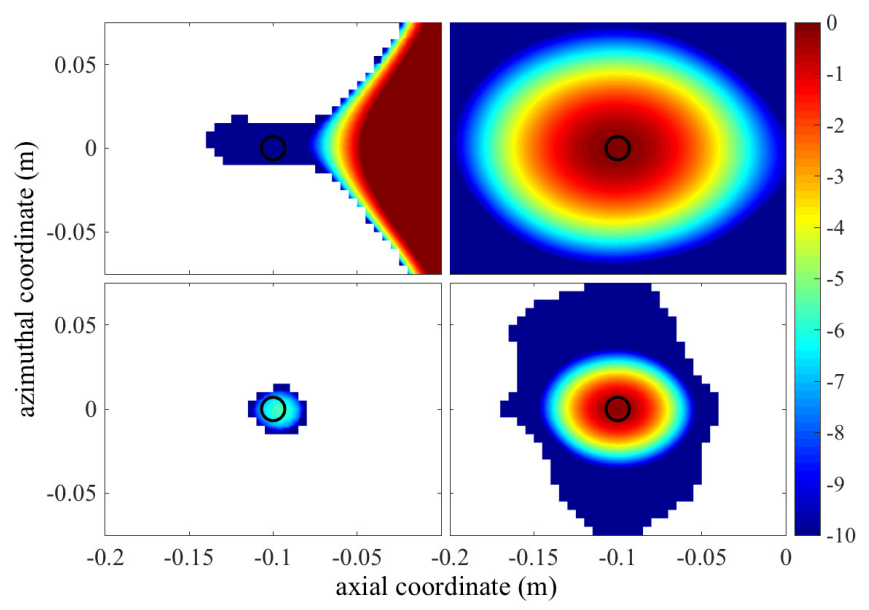

Fig. 9 Estimated spectrum levels, dB relative to true value, for secondary source in side-lobes. Left: ROSI; right: current method. Top: $2.4 \mathrm{kHz}$; bottom: $4.8 \mathrm{kHz}$. Circle indicates source position.

In contrast, the maps for the current method successfully identify both source position and level at each frequency. These results were generated with the primary-source position assumed to be correctly known (as can reasonably be anticipated, given its dominance), and that of the secondary varied. They clearly illustrate the benefit of solving for multiple sources simultaneously; effectively, the main-source side-lobes are eliminated, allowing the secondary source to be located without interference.

\section{Main-lobe interference}

The success of the current method in eliminating primary-source side-lobe interference from a secondary source estimate invites a further question: can it identify a secondary source within the main lobe of the primary? Hence the final example considered here has a secondary source $10 \mathrm{~dB}$ weaker than the primary, and offset from it by $0.063 \mathrm{~m}$ $(0.05 \mathrm{~m}$ in the axial direction and $0.038 \mathrm{~m}$ in the azimuthal). This means that, in the ROSI maps of Fig. 8 , it is below the main-lobe level at $2.4 \mathrm{kHz}$, but above it at $4.8 \mathrm{kHz}$. Unsurprisingly, then, it cannot be discerned by ROSI at the lower frequency; Fig. 10 (covering the same region as Fig. 8) shows that almost all the estimates in the map range lie above its true level. At $4.8 \mathrm{kHz}$, its effect is visible, but only as a distortion of the primary trace. Without prior knowledge, this plot would not provide convincing evidence for the presence of a secondary source.

Meanwhile, at the same frequency, the current method correctly identifies the secondary source, and with the correct level. It also appears to be successful at the lower frequency. Certainly, the estimated level at the source position is correct. Note, however, that there is no minimum between the secondary and primary sources. In fact, the estimate increases monotonically from one to the other, and a different choice of color range would thus make the secondary far less clear. This is because, at this proximity to the primary, an erroneous focus point for the secondary leads to significant errors in the primary's level estimate too, even though its position is specified correctly. Again, there are fundamental resolution constraints imposed by the array configuration that cannot be escaped.

In this case, however, iterative source identification could be effective; if the primary can be characterized well enough in a single-source calculation, its contributions to the cross-correlations can be subtracted, and the secondary then sought in further single-source estimates. The results from this approach are shown in Fig. 11] The improvement in 


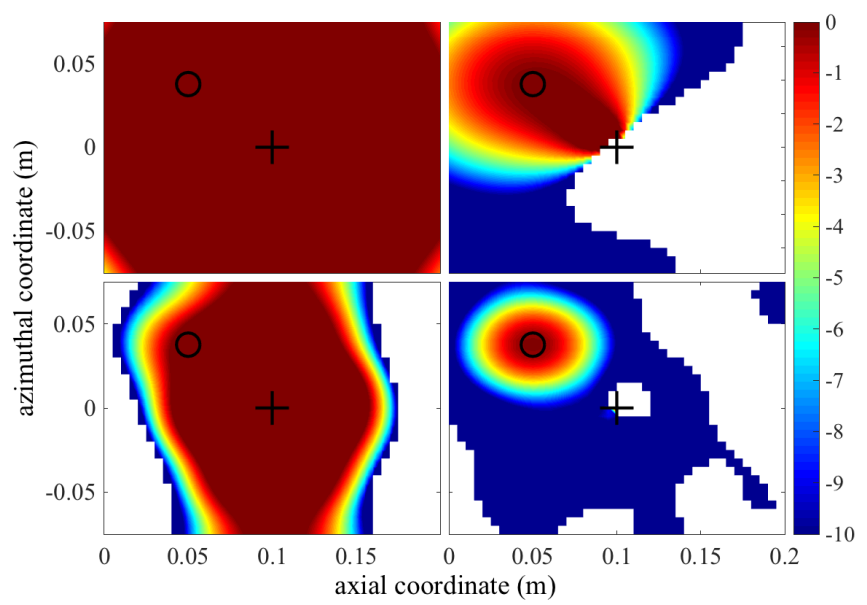

Fig. 10 Estimated spectrum levels, dB relative to true value, for secondary source near main lobe. Left: ROSI; right: current method. Top: $2.4 \mathrm{kHz}$; bottom: $4.8 \mathrm{kHz}$. Cross: primary source; circle: secondary.

discrimination at the lower frequency is evident: the secondary peak is now clearly isolated from the primary source. The price paid is some inaccuracy in the level estimate, because the single-source calculation for the primary is affected by contamination from the secondary. In this instance, the estimate for the secondary is $0.3 \mathrm{~dB}$ below the true value. This issue is, as one would expect, mitigated by increases in frequency; at $4.8 \mathrm{kHz}$ the level-estimate error is within statistical uncertainty.

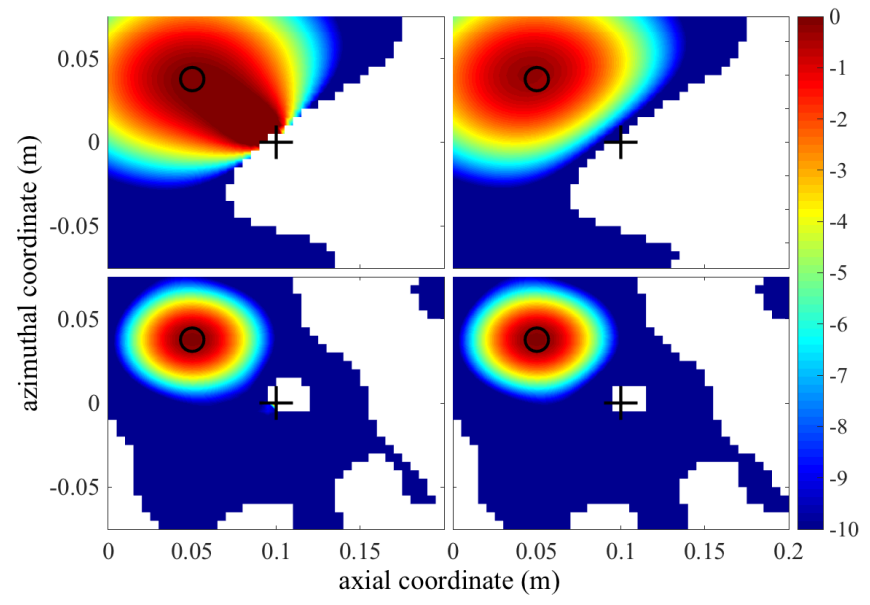

Fig. 11 Comparison of secondary-source level estimates from one-step (left) and iterated (right) calculations. Other details as Fig. 10 .

To summarize, the results of this section demonstrate the benefits of the current, multiple-source, formulation in two ways. At high enough frequencies, the main-lobe interference from a dominant source can effectively be eliminated to reveal the presence of a secondary contributor. At lower frequencies, when the array resolution is insufficient to sustain this ability, the secondary can still be found iteratively. Neither approach is possible with the ROSI method.

\section{G. Timing}

The results presented here were generated in the Matlab (R2018b) environment, running on a 2017 Apple iMac (3.6GHz Intel Core i7 processor, 16GB RAM). On the basis of elapsed time, the calculation and storage of the microphone cross-correlations takes approximately 11 minutes. For a single source, the auto-correlation fit requires $24 \mathrm{~s}$ 
per focus point; for the three-source case this increases to $92 \mathrm{~s}$. In comparison, the ROSI analyses take $96 \mathrm{~s}$ per focus point, almost all of which is occupied in signal de-Dopplerization.

\section{H. Discussion}

The results presented in this section have demonstrated the feasibility, and potential benefits, of the new method for one specific source/array configuration. Ultimately, its usefulness in general can only properly be established by subsequent application to other cases. However, it is possible to consider which aspects of the current simulations are necessary, and to what extent they may be encountered more widely.

The efficiency of Eq. (6) as a representation of the source auto-correlation is a crucial requirement; it reduces the set of unknown coefficients to a manageable size. Fortunately, this feature is likely to be robust. First, note that any deterministic tonal components linked to the rotation frequency can (and should) be removed in a pre-processing step. The remaining broadband sound, if it is an audible nuisance, is then typically of high bandwidth relative to the rotation frequency, with a smoothly varying spectrum. Hence it can be captured accurately with relatively low frequency resolution; i.e. a discrete representation would require only a few, wide, frequency 'bins'. This implies that the auto-correlation function will indeed be concentrated around $\tau_{m}=\tau_{n}$. Its variation with $\left(\tau_{m}+\tau_{n}\right) / 2$ will depend on the macroscopic flow conditions giving rise to the source. While these may not be steady, they usually change on a time-scale associated with the rotation period, as assumed. In any case, a dependence on $\left(\tau_{m}+\tau_{n}\right) / 2$ which is not slow cannot be accommodated by the ROSI approach either. (Recall that, if the dependence is slow, then the source can be approximated as statistically stationary over time windows long enough to obtain useful spectral estimates via ROSI.)

Next, consider the reduction in (receiver-time) lag range that was achievable here. This depends not only on the extent of the source auto-correlation, but also on the variation in receiver-time lag $\left(t_{n}-t_{m}\right)$ along the locus of maximum $R_{m n}$ (cf. Fig. 3). That variation scales approximately with $\ell / c$, where $\ell$ is the range spanned by the difference in source/microphone distances. Assuming that the array faces the noise-generating device (i.e. the separation vector is approximately normal to the array plane), $\ell$ diminishes with increasing distance between the two. Hence the worst case is when they are close, when $\ell$ is given by the smaller of the array size and the device size. The lag-range variation is to be compared with the rotation time, $t_{R}$. Thus significant reduction in receiver-time lag range relative to the nominal requirement is certainly achievable if the lag parameter, $\ell / c t_{R}$, is small, but may not be otherwise. Note, however, that this issue is not necessarily critical for the viability of the method, because it only affects the (one-off) calculation cost for the microphone cross-correlations and the off-line storage required to save the results. More important is the size of the equation set that must be solved for the source parameters.

Reducing the number of entries in each equation has already been addressed, via the source parameterization. The number of equations depends on how many cross-correlation samples correspond to a legitimate source auto-correlation lag value. As seen, it can be reduced by downsampling the $t_{m}$ dependence of $R_{m n}$. The degree of downsampling achievable depends on the spectral content of $R_{m n}$ along lines of fixed lag, $t_{n}-t_{m}$. This, in turn, depends on the angle at which the locus of maximum $R_{m n}$ crosses the lines. If it is parallel to them, the spectral content is set by the source auto-correlation variation at fixed source lag, $\tau_{n}-\tau_{m}$, and is thus low. As it departs from parallel, the spectral content increases. Hence the lag range spanned by the locus of maximum $R_{m n}$ is again relevant, but now in a sense that concerns viability (assuming that the calculation time for equation assembly and solution will become impracticably high if the set will not fit in available machine memory).

To summarize, it has been argued that the fundamental assumption made here - weak non-stationarity in the source auto-correlation function - should usually be legitimate in the applications envisaged. The method's viability will also depend on computational requirements. These will be configuration-specific, but some indication of their significance is given by the lag parameter $\ell / c t_{R}$.

Finally, if the current approach is applicable, should it be used instead of ROSI? There are two possible reasons for preferring it: explicit recognition of statistical non-stationarity in the source(s), and multi-source capability. The first is, however, not especially compelling, given that the non-stationarity is assumed to be weak and frequency-resolution requirements to be low. The significance of the second will depend on the situation. For well-separated sources of comparable strength, the combination of ROSI with an effective array geometry may well be satisfactory. However, if sources are clustered, or widely varying in strength, the cross-correlation-based method can identify features that are effectively invisible to ROSI, and thus should be considered. 


\section{Conclusion}

This paper has presented an alternative to the ROSI algorithm for rotating-source identification. It works with the cross-correlations of the sound measurements from such sources, rather than attempting to re-create the source signal by de-Dopplerizing each measurement. This formulation explicitly recognizes the non-stationarity of the measurements, and thereby also allows for sources which are not statistically stationary. More importantly, it permits identification of multiple sources in a single processing step, unlike the de-Dopplerization approach. Also implied, therefore, is the possibility of iterative source identification, in which successive calculations account for the contributions of sources already characterized. However, the new method makes significant, and potentially unrealistic, demands on computer memory and processing power.

These demands can be mitigated by a series of measures to reduce the size of the governing equation set. Given a weakly non-stationary source, it has been shown via a simulated example that the problem can be rendered solvable in a reasonable time (minutes, rather than hours) on a present-day desktop computer.

The simulations have also generated sample results for comparison against ROSI output. For a group of three identical rotating sources, the ROSI estimate of the individual source spectrum is up to $2 \mathrm{~dB}$ in error in the design frequency range of the array configuration employed. The current method exhibits similar error if it focuses on a single source, but (as expected) achieves the correct result when formulated to identify all three simultaneously. Spatial discrimination has also been tested, by generating beamforming maps over focus-point grids. Resolution — the capacity to locate a single source correctly — is broadly equivalent for the two methods. Secondary source detection in the presence of a stronger primary source is markedly better with the current approach. In an example where the primary-source side-lobes interfere significantly with the ROSI map around the secondary source, the accuracy of the cross-correlation-based method is unaffected. When the secondary source is close enough to the primary-source main lobe to be masked in the ROSI map, it is successfully identified. Finally, even when it lies within the main lobe, its presence can be revealed via an iterative approach.

The extent to which the new method is applicable in general remains to be determined. The need for any source non-stationarity to be weak is not a strong constraint; it is also a prerequisite for ROSI, and is likely to be satisfied in practice. A configuration giving rise to a manageable equation set, however, is a requirement only for the current method, and cannot necessarily be guaranteed. A crucial point here is the extent of the source auto-correlation function. The parameterization employed for its time-lag dependence has size proportional to the auto-correlation-function length. For the number of unknown coefficients to remain manageable, the auto-correlation must decay to zero over a time much shorter than the rotation period. This implies that the associated spectrum needs to be broadband and smooth, so that it can accurately be characterized with relatively low frequency resolution. Deterministic noise components at the rotation frequency and its harmonics are thus precluded, and need to be removed before the cross-correlations are formed. This is a straightforward step, as such components can be identified by phase-locked time-domain averaging of the measured microphone signals.

The restriction to broadband, stochastic, noise components means that the method proposed here would not be appropriate for assessing blade thickness noise, or the deterministic component of loading noise. It is, on the other hand, applicable in principle to stochastic loading noise (e.g. caused by blade/turbulence interactions) and turbulence noise, including broadband blade self-noise. Its feasibility in practice will depend on the the spectral frequency resolution demanded by the specific case under consideration.

\section{Appendix A. Signal synthesis}

A set of microphone signals corresponding to a known source spectrum is needed. This requires two steps: first, a stochastic source signal with the specified spectrum must be created; and second, the associated microphone signals must be calculated according to Eq. (11). The error due to the interpolation employed in the latter step should be negligible.

The issue of interpolation error in the acoustic context has been discussed by Graham [12]. A fundamental point is that a signal with significant spectral content up to its Nyquist frequency cannot realistically be interpolated without error. This implies that the simplest option for source simulation, uncorrelated samples (implying a flat spectrum), is not available; some form of low-pass filtering must also be applied. The degree of filtering required can be assessed by consideration of the proposed interpolation function's spectral content [12]. In particular, if septimic basis splines are used to interpolate a signal which is effectively band-limited to $24 \%$ of its sampling frequency, the resulting (continuous) interpolant will, for practical purposes, be similarly band-limited. (Out-of-band aliases will be over $80 \mathrm{~dB}$ below the signal level, and in-band attenuation will be less than $0.01 \%$.)

To generate such a signal, uncorrelated, unit-variance, Gaussian-distributed samples $s_{n}$ are passed through a 
finite-impulse-response filter with 'taps' $h_{n}$ to give

$$
\sigma(n T)=\sum_{m=1}^{M} h_{m} s_{n-m},
$$

where $T$ is the sample spacing. Corresponding to $\sigma(n T)$ is a unique, continuous, band-limited signal $\sigma(\tau)$ with spectrum defined by

$$
S_{\sigma \sigma}(\omega)=\int_{-\infty}^{\infty} \overline{\sigma(\tau) \sigma(\tau+\Delta \tau)} \mathrm{e}^{-\mathrm{i} \omega \Delta \tau} \mathrm{d}(\Delta \tau) .
$$

The continuous signal is linked to its discrete counterpart via the ideal 'sinc'-function interpolator [12], and this allows the spectrum to be expressed in terms of the filter coefficients:

$$
\begin{aligned}
S_{\sigma \sigma}(\omega) & =T\left|\sum_{m=1}^{M} h_{m} \mathrm{e}^{-\mathrm{i} m \omega T}\right|^{2}, \quad|\omega T|<\pi ; \\
& =0, \quad \text { otherwise. }
\end{aligned}
$$

Coefficients imposing the desired bandwidth limit on $S_{\sigma \sigma}(\omega)$ can be obtained via the classical 'windowed-sinc' approach to low-pass digital filter design [14]. In particular, the specification

$$
h_{m}=\frac{\sin [\pi(m-36) / 3]}{\pi(m-36)} \mathrm{e}^{-(m-36)^{2} / 225}, \quad m=1, \ldots, 71
$$

fulfils the requirement, giving spectral attenuation of at least $90 \mathrm{~dB}$ for frequencies above $24 \%$ of the sampling rate.

Having synthesized a uniformly spaced source signal according to Eq. [13, uniformly spaced microphone signals are obtained by first inverting Eq. (11) to find the source times (numerically, via Newton-Raphson iteration), then interrogating the source-signal interpolant at those times, and finally combining the results with the transmission factors given by Eq. (9). Note that the extremes of the source-signal sample set are unusable due to filtering and interpolation transients, and this constraint is respected in the choice of receiver times. Also, the limited bandwidth of the source signal implies that the microphone signals will be similarly restricted; even with the Doppler shift, they will contain negligible frequency components above $25 \%$ of the source-signal sampling rate. Hence they can be sampled at half the rate without aliasing, and this point is exploited to reduce storage requirements. Choosing the source-sample generation rate so that 2048 microphone-signal samples are taken per revolution then band-limits the source to below $11.8 \mathrm{kHz}$.

\section{Appendix B. Determination of cross-correlation cut-off order}

The potential for down-sampling the $t_{m}$ dependence of $R_{m n}\left(t_{m}, t_{n}-t_{m}\right)$ can only be fully realized if the filter cut-off frequency is tailored for each microphone pair. Hence a means of identifying the spectral transition from signal to noise is needed. For robustness and generality, it should require minimal user input.

The approach chosen is based on a threshold analysis of the spectrum (cf. Fig. 44). First, attention is restricted to the line from the mean of the lag range, because this exhibits the most rapid variation with $t_{m}$. (In Fig. 3 this choice corresponds to $t_{n}-t_{m}=-1.3 \mathrm{~ms}$.) Then, for successively lower threshold values, the highest order whose amplitude exceeds the threshold is identified. In the signal range, this quantity will be relatively small, and will increase slowly. Once the threshold descends to the noise region, it will rapidly jump towards the maximum order. This behavior is illustrated in Fig. 12, which shows the highest exceeding order plotted against threshold value for the example of Fig. 4

Next, the threshold-analysis results must be converted to a cut-off order. A simple and robust method is to specify a boundary value for the highest exceeding order, chosen so that it lies in the noise region for all microphone pairs. This is the only user input required; for the data sets considered here it is set at 99 . The microphone-pair cut-off order then comes from the threshold-analysis data point immediately below the boundary, and the number of orders used to generate the down-sampled signal is the next appropriate power of two. (The power-of-two requirement ensures that the new data points coincide with sample-time values for which the associated source times are stored. Its beneficial speed implications are not particularly important, given the relatively short discrete Fourier transforms involved.)

For the current example, the cut-off order is 51 and the down-sampled cross-correlation has 128 values of $t_{m}$. Figure 13 shows the result for the $t_{n}-t_{m}=-1.3 \mathrm{~ms}$ lag line. The down-sampled values do not always directly overlay the 


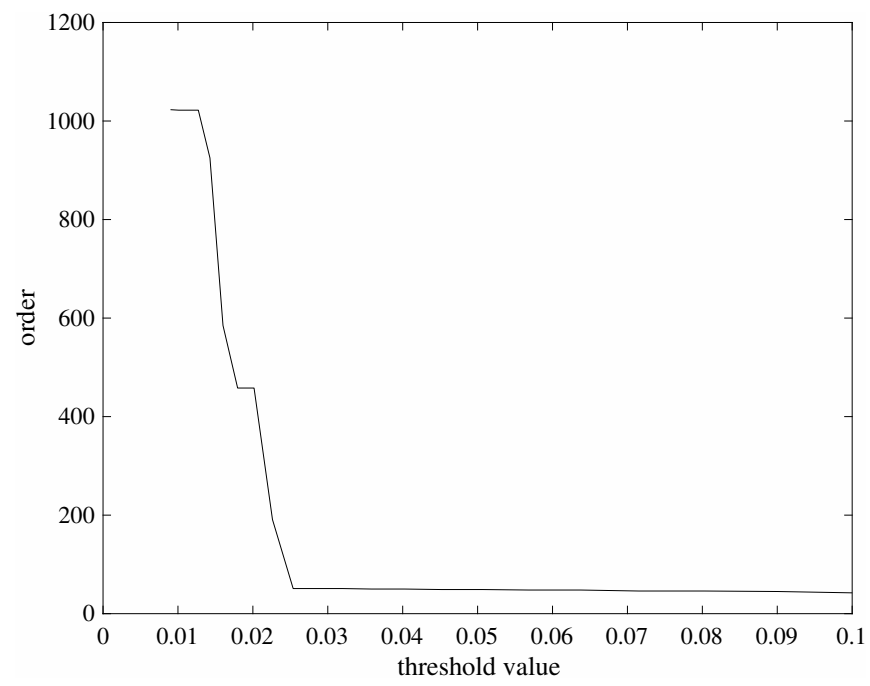

Fig. 12 Highest order with spectral magnitude exceeding given threshold values for the $t_{n}-t_{m}=-1.3 \mathrm{~ms}$ line in Fig. 3. Threshold-value steps are $1 \mathrm{~dB}$.

original data, but this is to be expected given the filtering employed. Their validity has been checked via comparison with an intermediate signal, generated from the filtered Fourier orders without any downsampling.

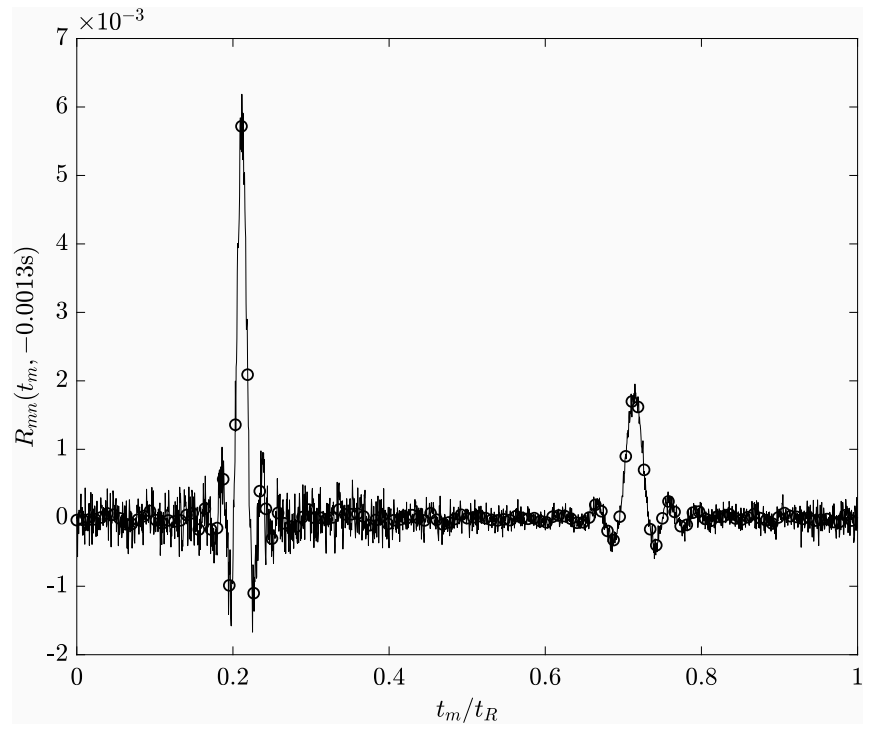

Fig. 13 The original (一) and down-sampled ( $\circ$ ) cross-correlations for the $t_{n}-t_{m}=-1.3 \mathrm{~ms}$ line in Fig. 3 .

\section{Acknowledgments}

This work was undertaken without external funding. The implicit financial support provided by the University of Cambridge is gratefully acknowledged. Supporting research data are available at: https://doi.org/10.17863/CAM.38304. 


\section{References}

[1] Soderman, P. T., and Allen, C. S., "Microphone measurements in and out of airstream," Aeroacoustic Measurements, edited by T. J. Mueller, Springer-Verlag, Berlin Heidelberg, 2002, Chap. 1.

[2] Underbrink, J. R., “Aeroacoustic phased array testing in low speed wind tunnels," Aeroacoustic Measurements, edited by T. J. Mueller, Springer-Verlag, Berlin Heidelberg, 2002, Chap. 3.

[3] Dougherty, R. P., "Beamforming in acoustic testing," Aeroacoustic Measurements, edited by T. J. Mueller, Springer-Verlag, Berlin Heidelberg, 2002, Chap. 2.

[4] Brooks, T. F., and Humphreys, W. M., "A deconvolution approach for the mapping of acoustic sources (DAMAS) determined from phased microphone arrays,” Journal of Sound and Vibration, Vol. 294, No. 4-5, 2006, pp. 856-879. doi:10.1016/j.jsv.2005.12.046.

[5] Sijtsma, P., “CLEAN based on spatial source coherence," International Journal of Aeroacoustics, Vol. 6, No. 4, 2007, pp. 357-374. doi:10.1260/147547207783359459.

[6] Howell, G. P., Bradley, A. J., McCormick, M. A., and Brown, J. D., "De-Dopplerization and acoustic imaging of aircraft flyover noise measurements," Journal of Sound and Vibration, Vol. 105, No. 1, 1986, pp. 151-167. doi:10.1016/0022-460X(86)90227-0.

[7] Sijtsma, P., Oerlemans, S., and Holthusen, H., "Location of rotating sources by phased array measurements," AIAA Paper 2001-2167, 2001. doi:10.2514/6.2001-2167.

[8] Sijtsma, P., "Beamforming on moving sources,” Tech. Rep. NLR-TP-2006-733, National Aerospace Laboratory NLR, 2007.

[9] Pearson, C., and Graham, W. R., "Experimental characterization of vertical-axis wind turbine noise," The Journal of the Acoustical Society of America, Vol. 137, No. 1, 2015, pp. EL111-EL116. doi:10.1121/1.4904915.

[10] Shin, H.-C., Graham, W. R., Sijtsma, P., Andreou, C., and Faszer, A. C., "Implementation of a phased microphone array in a closed-section wind tunnel,” AIAA Journal, Vol. 45, No. 12, 2007, pp. 2897-2909. doi:10.2514/1.30378.

[11] Hou, H., and Andrews, H., "Cubic splines for image interpolation and digital filtering," IEEE Transactions on Acoustics, Speech, and Signal Processing, Vol. 26, No. 6, 1978, pp. 508-517. doi:10.1109/TASSP.1978.1163154.

[12] Graham, W. R., "Interpolation for de-Dopplerisation,” Journal of Sound and Vibration, Vol. 422, 2018, pp. $210-236$. doi:10.1016/j.jsv.2018.02.002.

[13] Welch, P., "The use of fast Fourier transform for the estimation of power spectra: a method based on time averaging over short, modified periodograms," IEEE Transactions on Audio and Electroacoustics, Vol. 15, No. 2, 1967 , pp. 70-73. doi:10.1109/TAU.1967.1161901.

[14] Saramäki, T., "Finite impulse response filter design," Handbook for Digital Signal Processing, edited by S. K. Mitra and J. F. Kaiser, Wiley, New York, 1993, Chap. 4. 